\title{
ESTUDO E ANÁLISE DA MISTURA DE ÁGUA E ETANOL ATRAVÉS DE EQUAÇÕES DE ESTADO.
}

\author{
S. F. VASCONCELOS ${ }^{1}$, F. J. F. CHAVES ${ }^{1}$, C. V. FERNANDES ${ }^{1}$, N. SILVA ${ }^{1}$, H \\ $\mathrm{BISPO}^{1}$ \\ ${ }^{1}$ Universidade Federal de Campina Grande, Unidade Acadêmica de Engenharia \\ Química \\ E-mail para contato: helenobispo@deq.ufcg.edu.br
}

\begin{abstract}
RESUMO - A predição de propriedades termodinâmicas de processos é de grande interesse da indústria química. De um modo geral, estudos envolvendo equilíbrio de fase em sistemas binários podem ser modelados através de equações de estado e aplicados as condições de operação com o intuito de desenvolvimento e/ou otimização termodinâmica dos processos. A equação de estado de Peng-Robinson foi utilizada para a previsão do comportamento de uma mistura de água e etanol. Os resultados obtidos foram comparados com os resultados gerados pela plataforma de simulação Aspen Plus e os adquiridos experimentalmente em laboratório. A análise dos resultados indica uma boa concordância com os valores experimentais e os fornecidos pela literatura, indicando que os softwares foram capazes de prever qualitativamente o comportamento do sistema binário.
\end{abstract}

\section{INTRODUÇÃO}

A definição do volume de mistura é um parâmetro de grande importância para a indústria química. Tal informação deve ser muito bem calculada para que os projetos de equipamentos e processos possam ser conduzidos de forma satisfatória. Devido à diferença das forças intermoleculares entre os componentes em uma solução, a medida do volume total resultante da mistura líquidos reais desvia do volume total, quando calculado a partir dos volumes das espécies individuais (contração/expansão de volume), conhecido termodinamicamente como volume de excesso. Para que este comportamento não ideal possa ser matematicamente observado, define-se uma classe de propriedades termodinâmicas, conhecida por propriedade parcial molar, mais especificamente o volume parcial molar. Tais propriedades são dependentes da composição do sistema, podendo ser determinada experimentalmente.

As quantidades parciais molares definem como uma determinada propriedade extensiva de uma solução varia com a composição molar mistura, ou seja, com adição ou remoção infinitesimal de um dado componente da solução a uma temperatura e pressão constantes. Dentre as propriedades parciais, o volume parcial molar é a quantidade mais fácil de ser visualizada, sendo esta definida como a contribuição volumétrica que um componente tem para o volume total da solução. Sendo assim, o volume parcial molar de uma substância A, em um mistura, é a variação de volume total da mistura provocada pela adição de um mol de A (ATKINS, 1999). Desse, este 
trabalho tem por objetivo analisar o comportamento do volume parcial molar em um sistema composto por uma solução de etanol e água em escala laboratórial, e utilizar os dados obtidos como referência em um estudo envolvendo diferentes equações de estado em uma plataforma de simualçao (Aspen Plus) para que tal volume possa ser previsto. Os resultados obtidos apresentam uma boa concordância com o experimento prático, demonstrando que a plataforma de simulação pode ser utilizada para a previsão do comportamento das propriedades parciais em processos químicos.

\section{FUNDAMENTAÇÃO TEÓRICA}

Os volumes parciais molares dos componentes de uma mistura variam de acordo com a composição, tendo em vista que as vizinhanças das moléculas se alteram a medida que a composição de um determinado componente passa a integrar e interagir com um outro componente antes puro (ATKINS, 1999). Tal modificação do ambiente resulta em uma variação das forças intermoleculares que agem entre as moléculas, sendo esta a responsável pela variação das propriedades extensivas de uma solução.

Sendo assim, pode-se denominar por $\mathrm{X}$ uma grandeza termodinâmica extensiva qualquer (U, H, V, etc.), sua diferencial total, em termos de temperatura (T), pressão (P) e quantidade de substância (n) presente no sistema, deverá ser escrita na forma mais geral como:

$d X=\left(\frac{\partial X}{\partial T}\right)_{P, n} d T+\left(\frac{\partial X}{\partial P}\right)_{T, n} d P+\sum_{i}^{n}\left(\frac{\partial X}{\partial n_{i}}\right)_{T, P, n_{j}} d n_{i}$

onde $n$ é a quantidade de substância do componente, e os subscritos i e j representam os componentes presentes na solução. Já o termo $\frac{\partial X}{\partial n_{i}}=\bar{X}_{\mathrm{i}}$ é conhecido como grandeza ou propriedade molar parcial.

Desse modo, pode-se definir o volume parcial molar de um determinando componente em uma composição conhecida como:

$V_{j}=\left(\frac{\partial V}{\partial n_{j}}\right)_{P, T, n}$

onde $n_{j}$ representa o número de mols do componente $j$.

Então pode afirmar que o volume parcial molar é o coeficiente angular da curva do volume total da mistura em função dos moles de um dado componente (KORETSKY, 2007), mantendo-se composição dos outros elementos constantes.

Se a composição de uma mistura for alterada pela adição infinitesimal de mols de $\mathrm{A}, \mathrm{d} \boldsymbol{n}_{\mathrm{A}}$, e mols de $\mathrm{B}, \mathrm{d} \boldsymbol{n}_{\mathrm{B}}, \mathrm{o}$ volume total da mistura sofrerá uma alteração infinitesimal, $\mathrm{dV}$, dada por: 
$d V_{=}\left(\frac{\partial V_{r}}{\partial n_{A}}\right)_{P, T, n_{B}} d x_{A}+\left(\frac{\partial V_{r}}{\partial n_{A}}\right)_{P, T, n_{A}} d x_{B}$

$d V=\overline{V_{A}} d x_{A}+\overline{V_{B}} d x_{B}$

Uma vez conhecidos os volumes parciais molares dos componentes de uma mistura binária, na composição de interesse, pode-se determinar o volume total da mistura real:

$V_{r}=\overline{V_{A}} x_{A}+\overline{V_{B}} x_{B}$

Dessa forma, pode-se definir o volume molar médio da mistura $\left(\Delta_{M} V\right)$ como a diferença entre o volume real da mistura e o seu volume ideal, ou seja, da substância pura.

$\Delta_{M} V=V_{r}-V_{i d}$

onde $V_{i d}$ é dado por:

$V_{i d}=\mathrm{V}_{\mathrm{A}} x_{A}+\mathrm{V}_{\mathrm{B}} x_{B}$

Substituindo as equações (5) e (7) na equação (7) e sabendo que $x_{A}=1-x_{B}$, tem-se:

$\Delta_{M} V=\left[\left(\overline{V_{A}}-\overline{V_{B}}\right)-\left(\mathrm{V}_{\mathrm{A}}-\mathrm{V}_{\mathrm{B}}\right)\right] x_{A}+\left(\overline{\mathrm{V}}_{\mathrm{B}}-\mathrm{V}_{\mathrm{B}}\right)$

Logo,

$\frac{d\left(\Delta_{M} V\right)}{d x_{A}}=\frac{d V_{r}}{d x_{A}}=\frac{d V_{i d}}{d x_{A}}=\left(\overline{V_{A}}-\overline{V_{B}}\right)-\left(\mathrm{V}_{\mathrm{A}}-\mathrm{V}_{\mathrm{B}}\right)$

Substituindo os respectivos valores para A e B obtém-se as seguintes relações:

$\overline{V_{A}}=\Delta_{M} V-\frac{d\left(\Delta_{M} V\right)}{d x_{A}} x_{B}+\mathrm{V}_{\mathrm{A}}$

$\overline{V_{B}}=\Delta_{M} V-\frac{d\left(\Delta_{M} V\right)}{d x_{A}} x_{A}+V_{B}$

De acordo com as relações acima, os volumes molares parciais dos componentes A e B para as composições específicas $\left(x_{A}, x_{B}\right)$ podem ser determinados se os volumes 
molares das substâncias puras $\left(V_{A}, V_{B}\right)$ e seus quocientes diferenciais $\frac{d\left(\Delta_{M} V\right)}{d x_{A}}$ forem conhecidos. Isto pode ser obtido como a tangente do gráfico que relaciona $\Delta_{M} V$ e $x_{A}$, como mostra a Figura 1 abaixo. Porém a relação pode ser melhor calculada derivandose a correlação funcional $\Delta_{M} V=f\left(x_{A}\right)$ para valores selecionados de $x_{A}$.

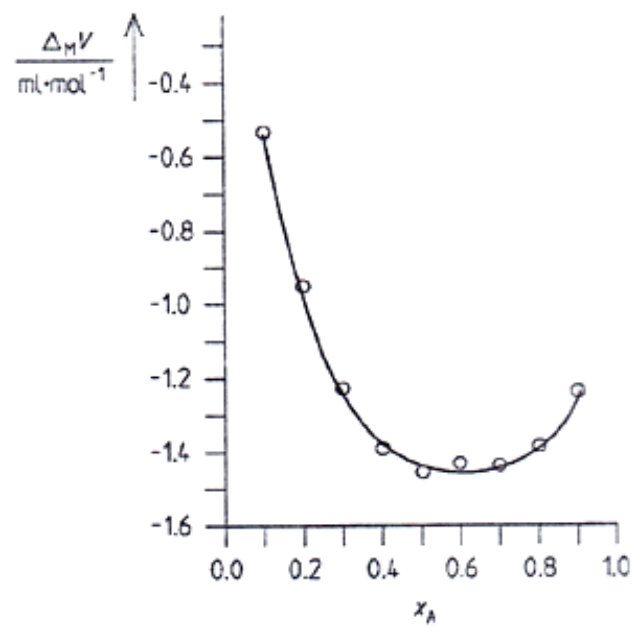

Figura 1. Dependência do volume molar médio da mistura $\Delta_{\mathrm{M}} \mathrm{V}$ sobre a composição de diferentes misturas de Etanol e Água descritas pela fração molar de Etanol a $20^{\circ} \mathrm{C}$.

Com o objetivo de responder com exatidão ao comportamento soluções ideais, têm-se as equações de estado cúbicas que são na realidade, as equações mais simples capazes de representar o comportamento termodinâmico dos sistemas, as quais apresentam um compromisso com a generalidade e simplicidade, sendo este adequado a muitas aplicações industriais, como por exemplo a indústria de bebidas (SMITH et al, 2007).

Sabendo-se que uma equação de estado é definida como uma relação entre as propriedades de pressão, temperatura e volume molar de uma substância através de relação matemática entre as grandezas termodinâmicas e as funções de estado de um dado sistema termodinâmico, pode-se, por meio de modelagem matemática representar o comportamento de propriedades, tais como entalpia (h), entropia (s), volume molar $(\mathrm{v})$, calor específico isobárico $\left(\mathrm{c}_{\mathrm{p}}\right)$, dentre outras propriedades termodinâmicas correlatas, as quais são extremamente úteis para a descrição de vários processos (LEAL, 2012).

Devido a restrições operacionais, a equação ideal não se aplica a condições mais severas de pressão e temperatura. Tal fato conduz ao desvio da idealidade, uma vez que o volume ocupado em uma solução varia devido as interações intermoleculares entre as moléculas das substâncias constituintes da solução, tornando a equação ideal incapaz de fornecer uma representação válida da realidade. Contudo, vários estudos foram propostos para corrigir tal problema, sendo desenvolvidas sucessivas equações de estado com modificações substanciais. 
Nesta perspectiva, Peng e Robinson, em 1976, propuseram uma equação de estado que foi considerada a mais bem sucedida em relação as anteriores, apresentando um melhor desempenho, sobretudo nas vizinhanças do ponto crítico e para cálculos de densidade de líquido. A equação Peng-Robinson, Equação 12, pode ser aplicada em cálculos de propriedades termodinâmicas de fluidos puros e misturas.

Além disso, está entre as equações de estado de uso mais disseminado para simulação e modelagem termodinâmica de processos industriais, devido a sua eficiência, simplicidade e facilidade de solução (LEAL, 2012).

$P=\frac{R T}{V_{m}-b}-\frac{a \propto}{V_{m}^{2}+2 V_{m}-b^{2}}$

onde os coeficientes presentes são dados por: $a=\frac{0.45724 R^{2} T_{c}^{2}}{P_{c}}, b=\frac{0.07780 R T_{c}}{P_{c}} \mathrm{e}$ $\propto=\left(1+\left(0,37464+1.54226 w-0,26992 w^{2}\right)\left(1-T_{r}^{0.5}\right)\right)^{2} ;$

\section{MATERIAIS E MÉTODOS}

Com o intuito de se obter o volume parcial molar de uma solução de etanol e água em diferentes composições, o seguinte experimento foi realizado, baseando-se nos princípios termodinâmicos clássicos, proporcionando assim a possibilidade de avaliação o comportamento ideal e não ideal da solução. O experimento foi conduzido em diferentes temperaturas, de acordo com a Figura 2 abaixo:

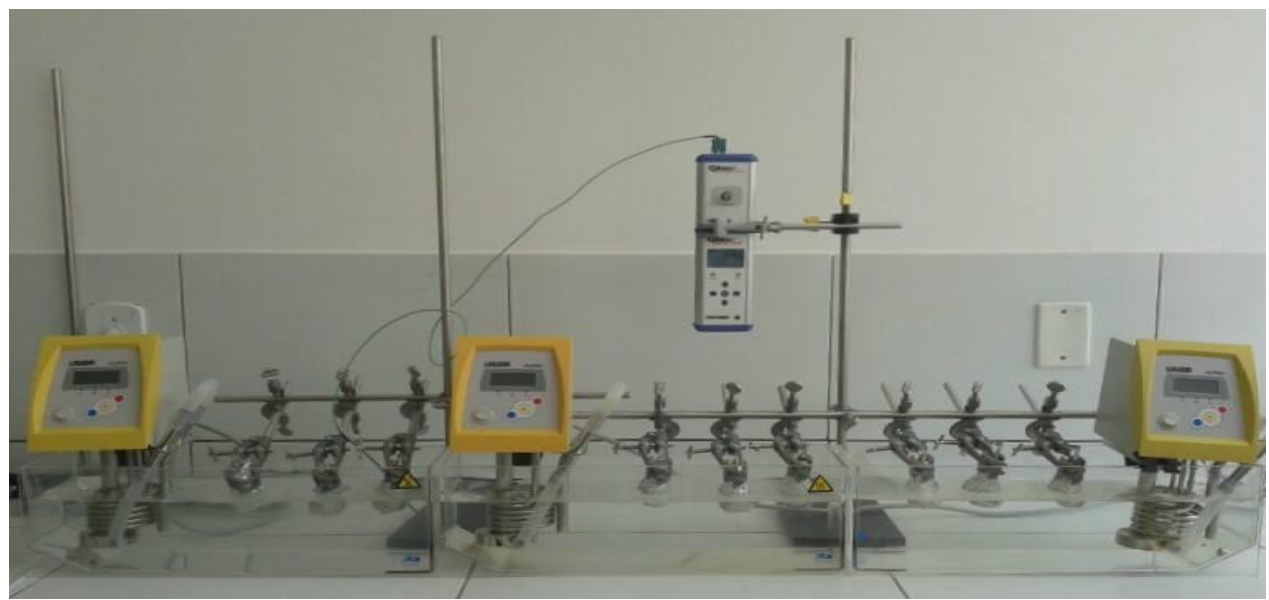

Figura 2 - Experimento para avaliação do volume parcial molar da solução binária de Etanol/Água

Para uma comparação entre os dados experimentais obtidos a plataforma de simulação Aspen Plus foi utilizada, como mostra a Figura 3 abaixo. O modelo desenvolvido é composto por uma bomba centrífuga e as correntes materiais, utilizando as composições do experimento apresentado pela Figura 2. O teste realizado utilizou as principais equações de estado, a saber: Soave-Redlich-Kwong (SRK), Peng e Robinson (PR) e a Lei dos Gases Ideais. 


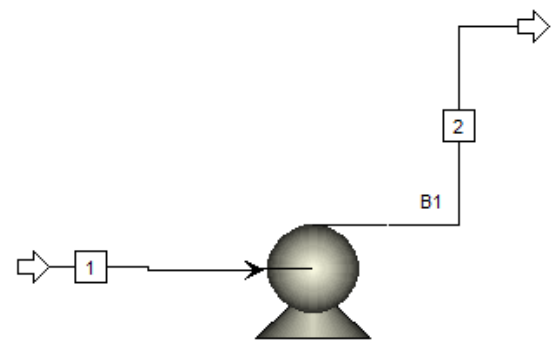

Figura 3 - Flowsheet do processo no Aspen Plus®

\section{RESULTADOS E DISCUSSÃO}

Os resultados obtidos a partir do experimento são mostrados na Tabela 1.

\begin{tabular}{ccc|cc|cc|c}
\hline $\begin{array}{c}\text { Composição } \\
\text { Molar de } \\
\text { Etanol }\end{array}$ & $\begin{array}{c}\text { Composição } \\
\text { Molar de } \\
\text { Água }\end{array}$ & \multicolumn{2}{c}{$\begin{array}{c}\text { Volume Molar } \\
\text { Real da mistura } \\
(\mathbf{m l} / \mathbf{m o l})\end{array}$} & \multicolumn{2}{c}{$\begin{array}{c}\text { Volume Molar } \\
\text { Ideal da } \\
\text { mistura } \\
(\mathbf{m l} / \mathbf{m o l})\end{array}$} & \multicolumn{2}{c}{$\begin{array}{c}\text { Variação do } \\
\text { Volume Molar da } \\
\text { mistura (ml/mol) }\end{array}$} \\
\cline { 3 - 8 } & & $20^{\circ} \mathrm{C}$ & $30^{\circ} \mathrm{C}$ & $20^{\circ} \mathrm{C}$ & $30^{\circ} \mathrm{C}$ & $20^{\circ} \mathrm{C}$ & $30^{\circ} \mathrm{C}$ \\
\hline 0,899 & 0,101 & 53,672 & 54,338 & 54,192 & 54,856 & $-0,520$ & $-0,518$ \\
\hline 0,800 & 0,200 & 49,277 & 50,039 & 50,227 & 50,819 & $-0,950$ & $-0,780$ \\
\hline 0,688 & 0,312 & 44,493 & 44,962 & 45,713 & 46,222 & $-1,220$ & $-1,260$ \\
\hline 0,595 & 0,405 & 40,585 & 41,370 & 41,985 & 42,427 & $-1,400$ & $-1,057$ \\
\hline 0,496 & 0,504 & 36,512 & 37,199 & 38,012 & 38,382 & $-1,500$ & $-1,183$ \\
\hline 0,398 & 0,602 & 32,602 & 33,266 & 34,052 & 34,351 & $-1,450$ & $-1,084$ \\
\hline 0,294 & 0,706 & 28,418 & 29,262 & 29,878 & 30,100 & $-1,460$ & $-0,839$ \\
\hline 0,199 & 0,801 & 24,684 & 25,363 & 26,084 & 26,238 & $-1,400$ & $-0,875$ \\
\hline 0,101 & 0,899 & 20,933 & 21,691 & 22,133 & 22,215 & $-1,200$ & $-0,524$ \\
\hline
\end{tabular}

Tabela 1 - Resultados do experimento realizado as temperaturas de $20^{\circ} \mathrm{C}$ e $30^{\circ} \mathrm{C}$

Como se pode observar os valores para a variação do volume molar da mistura, em ambas as temperaturas, são negativos, demonstrando assim que a solução sofre um processo de contração entre as moléculas. As variações do volume molar da mistura, volume de excesso, são apresentadas graficamente pelas Figuras 1 e 2, para as temperaturas de operação adotadas no experimento laboratorial, onde $\mathrm{T} 1=20^{\circ} \mathrm{C}$ e $\mathrm{T} 2=$ $30^{\circ} \mathrm{C}$. 


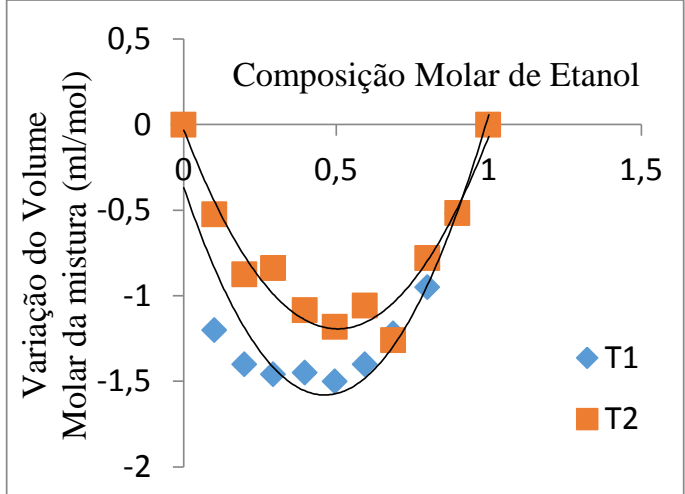

Figura 4 - Volume em excesso em função da composição molar de etanol

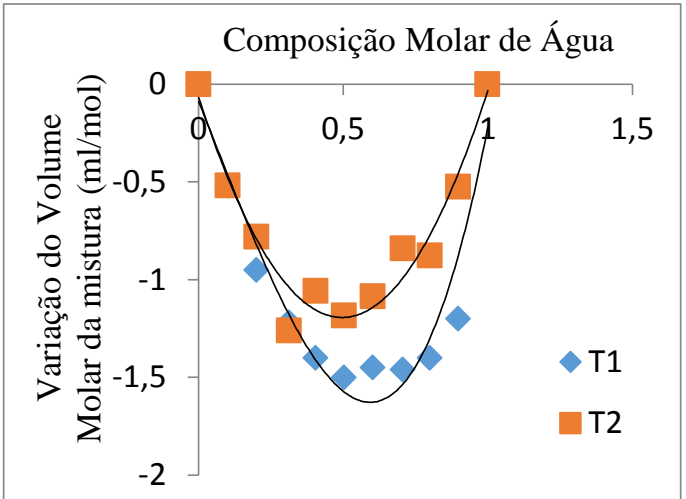

Figura 5 - Volume em excesso em função da composição molar de água

Através da simulação na plataforma Aspen Plus®, o volume molar real da mistura $(\mathrm{ml} / \mathrm{mol})$ pode ser obtido para cada temperatura, sendo ainda utilizadas as equações de estado anteriormente citadas, bem como as composições utilizadas no experimento laboratorial. Tais informações são sumarizadas nas Tabelas 3 e 4 abaixo.

\begin{tabular}{cccccccccc}
\hline \multirow{2}{*}{ Eq. } & \multicolumn{10}{c}{ Composição } \\
\cline { 2 - 10 } & $1^{\mathrm{a}}$ & $2^{\mathrm{a}}$ & $3^{\mathrm{a}}$ & $4^{\mathrm{a}}$ & $5^{\mathbf{a}}$ & $6^{\mathbf{a}}$ & $7^{\mathrm{a}}$ & $8^{\mathbf{a}}$ & $9^{\mathbf{a}}$ \\
\hline PR & 53,343 & 49,416 & 44,988 & 41,323 & 37,430 & 33,586 & 29,513 & 25,799 & 21,977 \\
\hline SRK & 53,343 & 49,416 & 44,988 & 41,323 & 37,430 & 33,586 & 29,513 & 25,799 & 21,977 \\
\hline LGI & 53,343 & 49,416 & 44,988 & 41,323 & 37,430 & 33,586 & 29,513 & 25,799 & 21,977 \\
\hline \multicolumn{1}{l}{ Tabela 3-Resultados o volume molar real da mistura (ml/mol) na temperatura de $21^{\circ} \mathrm{C}$}
\end{tabular}

\begin{tabular}{cccccccccc}
\hline \multirow{2}{*}{ Eq. } & \multicolumn{10}{c}{ Composição } \\
\cline { 2 - 10 } & $1^{\mathrm{a}}$ & $2^{\mathrm{a}}$ & $3^{\mathrm{a}}$ & $4^{\mathrm{a}}$ & $5^{\mathrm{a}}$ & $6^{\mathrm{a}}$ & $7^{\mathrm{a}}$ & $8^{\mathbf{a}}$ & $9^{\mathbf{a}}$ \\
\hline PR & 54,036 & 50,051 & 45,557 & 41,837 & 37,886 & 33,982 & 29,847 & 26,076 & 22,195 \\
\hline SRK & 54,036 & 50,051 & 45,557 & 41,837 & 37,886 & 33,982 & 29,847 & 26,076 & 22,195 \\
\hline LGI & 54,036 & 50,051 & 45,557 & 41,837 & 37,886 & 33,982 & 29,847 & 26,076 & 22,195 \\
\hline
\end{tabular}

Tabela 4 - Resultados o volume molar real da mistura $(\mathrm{ml} / \mathrm{mol})$ na temperatura de $30^{\circ} \mathrm{C}$

De um modo geral, os resultados obtidos para as equações de estado foram semelhantes, devido às condições operacionais impostas, ou seja, baixas pressões, sendo assim, próximos idealidade. Contudo, os dados experimentais foram comparados com os resultados das simulações, e o erro relativo entre eles foi calculado, como mostra a Tabela 5 abaixo.

\begin{tabular}{ccc}
\hline \multirow{2}{*}{ Composição } & \multicolumn{2}{c}{ Erro Relativo (\%) } \\
\cline { 2 - 3 } & $20^{\circ} \mathrm{C}$ & $30^{\circ} \mathrm{C}$ \\
\hline $\mathbf{1}^{\mathbf{a}}$ & 0,617796 & 0,558547 \\
\hline $\mathbf{2}^{\mathbf{a}}$ & 0,279755 & 0,024699 \\
\hline $\mathbf{3}^{\mathbf{a}}$ & 1,101008 & 1,307051 \\
\hline $\mathbf{4}^{\mathbf{a}}$ & 1,784687 & 1,116728 \\
\hline $\mathbf{5}^{\mathbf{a}}$ & 2,454009 & 1,811698 \\
\hline
\end{tabular}




\begin{tabular}{rrr}
\hline $\mathbf{6}^{\mathbf{a}}$ & 2,928194 & 2,107081 \\
\hline $\mathbf{7}^{\mathbf{a}}$ & 3,711875 & 1,962463 \\
\hline $\mathbf{8}^{\mathbf{a}}$ & 4,325391 & 2,736867 \\
\hline $\mathbf{9}^{\mathbf{a}}$ & 4,750195 & 2,268193
\end{tabular}

Tabela 5 - Erro Relativo entre os resultados obtidos pelo experimento laboratorial e a simulação na plataforma de simulação Aspen Plus®.

\section{CONCLUSÃO}

O estudo e a análise da mistura de água e etanol através das equações de estado, foi desenvolvido com base de que, em geral os volumes em excesso depende principalmente de dois efeitos, a modificação do ambiente que resulta em uma variação das forças intermoleculares quando dois componentes entram em contato; e da composição, pois as vizinhanças de cada tipo de molécula se alteram a medida que a composição passa de água pura para a de etanol puro. Foi observado que para cada equação de estado, Peng-Robinson, Soave-Redlich-Kwon e a Lei dos Gases Ideais, os mesmos resultados foram obtidos, isso se deve ao fato de que o experimento ocorreu próximo do comportamento ideal. O erro percentual relativo (E\%) no cálculo do volume especifico é pequeno e pode ser considerado como um erro sistemático devido as condições impostas no desenvolvimento do experimento. Percebeu-se também, durante o estudo, que cada equação tem sua peculiaridade e que dependendo das condições de operação, qualquer uma pode fornecer o valor mais próximo do verdadeiro.

\section{REFERÊNCIAS BIBLIOGRÁFICAS}

ATKINS, P. W. Físico-química. 6. ed. Rio de Janeiro: LTC, 1999.

SMITH, J.M. \& VAN NESS, H.C. Introduction to Chemical Engineering Thermodynamics. 4th Ed. New York, McGraw-Hill, Inc., 1987.

Koretsky M . D. Termodinâmica Para Engenharia Química. 1 Ed, Rio de Janeiro: LTC, 2007.

LEAL, D. S. Avaliação das equações de estado no cálculo de propriedades do dióxido de carbono úteis no estudo do seu escoamento. UFBA, 2012. Disponível em: http://www.pei.ufba.br/novo/uploads/defesas1/LEAL_PEI_DISSERTACAO_2012_apr ovada.pdf - Acessado em 20 de fevereiro de 2014. 